\title{
An Incremental Test to Identify the Pain Threshold Speed in Patients With Intermittent Claudication
}

\author{
Fabio Manfredini, MD; Cristina Mangolini, MD; Francesco Mascoli, MD*; \\ Gianni Mazzoni, MD; Maria Cristina Taddia, MD*; \\ Roberto Manfredini, MD**; Francesco Conconi, MD
}

\begin{abstract}
The purpose of this study was to develop a test for identifying the speed of onset of claudication, or pain threshold speed (PTS), in 16 patients affected by intermittent claudication. An echo-Doppler examination and the ankle-brachial index (ABI) determination were also performed. Test repeatability was evaluated in 10 patients retested within a few days. All 16 patients underwent the incremental walking test 3 times during a 6-month rehabilitation training program to verify the test's sensitivity in detecting the expected functional modifications. PTS was identified in all patients examined and the test-retest correlation coefficient (R) for PTS was 0.98. During the 6-month rehabilitation period, the ABI rose from $0.43 \pm 0.16$ to $0.72 \pm 0.15$ for the worst limb and PTS also rose significantly from $3.9 \pm 1.4 \mathrm{~km} / \mathrm{h}$ to $6.1 \pm 1.1 \mathrm{~km} / \mathrm{h}$. The average increments of ABI and PTS were significantly correlated. An incremental walking test for the identification of the walking speed at which claudication occurs has been developed. The PTS is a reproducible parameter that can be combined with other test results to establish the severity of the disease and to check any modifications that occur during rehabilitation. (Circ J 2002; 66: $1124-1127$ )
\end{abstract}

Key Words: Arteriopathy; Intermittent claudication; Rehabilitation; Walking test

C

hronic obliterative arteriopathy of the lower limbs is a widespread disabling pathology ${ }^{1}$ and a typical symptom is the appearance on walking of painful cramping called 'intermittent claudication'. This symptom depends on a discrepancy between oxygen supply, limited by the arteriopathy, and oxygen demand by the muscles active in walking? Claudication should therefore appear at a definite walking speed, varying from patient to patient according to the degree of arteriopathy and the development of collaterals.

The main purpose of this study was to identify the walking speed at which claudication occurs ("pain threshold speed' [PTS]), an additional parameter to be used in evaluating the severity of the disease. A secondary objective was to establish the practical use of PTS determination in identifying performance variations associated with rehabilitation programs.

\section{Methods}

\section{Patients}

Sixteen patients ( 12 males, 4 females) affected by peripheral arteriopathy in Fontaine's second stage of classification, were informed about the methods and purpose of the experimental protocol and agreed to participate in the

(Received May 14, 2002; revised manuscript received August 27, 2002; accepted September 18, 2002)

Centro Studi Biomedici Applicati allo Sport, Università di Ferrara, *Dipartimento di Chirurgia, Unità operativa di Chirurgia vascolare. Arcispedale S. Anna, Azienda Ospedale-Università di Ferrara and **Dipartimento di Medicina Clinica e Sperimentale, Sezione di Medicina, Interna I, Università di Ferrara, Italia

Mailing address: Fabio Manfredini, MD, Centro Studi Biomedici Applicati allo Sport, Università di Ferrara, Via Gramicia 35, I-44100,

Ferrara, Italy. E-mail: mdf@unife.it study. The experimental procedures were in accordance with the Ethical Standards of the Committee on Human Experimentation of the University. Admission to the study group was after approval by each patient's physician.

Following completion of a case-history form, clinical examination, and evaluation of the various tests performed (some patients had also undergone angiography), the subjects were started on the rehabilitation program. Of the 16 subjects who presented with chronic claudication of at least 6 months duration, 5 had undergone vascular surgery. All had been treated for several months with anticoagulants and 8 patients were also taking vasodilators because of the severity of their claudication. No modifications were made to any patient's therapies during the study period. The general characteristics of the 16 patients are listed in Table 1.

\section{Haemodynamic Measurements of the Lower Limbs}

The haemodynamic flow of the lower limb arteries (superficial and deep common femoral, popliteal, tibialis posterior, dorsalis pedis, peroneal) was measured in each patient by a bilateral echo-Doppler test (ACUSON 128/XP 10 'A.R.T.'), performed after the patient had rested in a supine position for at least $10 \mathrm{~min}$. The ratio between the systolic pressures recorded bilaterally at the level of the tibialis and brachial arteries (ankle-brachial index, ABI) was also determined. The ABI of the 2 legs were named worse $\mathrm{ABI}$ (wABI) and better $\mathrm{ABI}(\mathrm{bABI})$, depending on the values recorded.

\section{Determination of 'Pain Threshold Speed'}

The patients were submitted to an incremental walking test performed in the morning, during autumn and winter, in a heated corridor (ambient temperature $19-22^{\circ} \mathrm{C}$ ). The course, consisting of two 70-m straight sections connected by two $10-\mathrm{m}$ curves, was subdivided by markers into 16 
Table 1 General Characteristics of the Claudication Patients

\begin{tabular}{|c|c|c|c|c|}
\hline $\begin{array}{l}\text { Patient } \\
\text { (no. and sex) }\end{array}$ & $\begin{array}{c}\text { Age } \\
\text { (years) }\end{array}$ & $\begin{array}{l}\text { Weight } \\
(\mathrm{kg})\end{array}$ & $\begin{array}{l}\text { Risk factors } \\
\quad(*)\end{array}$ & $\begin{array}{c}\text { Arteriopathy } \\
\text { (major location) }\end{array}$ \\
\hline $1(F)$ & 39 & 58.5 & $S$ & Tibial \\
\hline $2(M)$ & 62 & 62.9 & $D, S$ & Popliteal and tibial \\
\hline $3(M)$ & 59 & 80.1 & $S$ & Femoral \\
\hline $4(M)$ & 68 & 70.5 & $H, D, S$ & Femoral and tibial \\
\hline $5(M)$ & 69 & 81.1 & $H, S$ & Iliac and femoral \\
\hline $6(M)$ & 65 & 69.2 & $D, S$ & Iliac and femoral \\
\hline $7(M)$ & 68 & 74.5 & $S$ & Tibial \\
\hline $8(M)$ & 56 & 74.1 & $D, S$ & Iliac \\
\hline $9(M)$ & 68 & 70.8 & $S$ & Femoral and popliteal \\
\hline $10(F)$ & 59 & 65.1 & $D, S$ & Iliac and femoral \\
\hline $11(F)$ & 53 & 80.2 & $S$ & Tibial \\
\hline $12(M)$ & 65 & 86.9 & $S$ & Iliac and femoral \\
\hline $13(M)$ & 72 & 89.5 & $S$ & Femoral \\
\hline $14(F)$ & 71 & 81.9 & $S$ & Femoral \\
\hline $15(M)$ & 51 & 62.8 & $H, D, S$ & Popliteal \\
\hline $16(M)$ & 69 & 87.1 & $S$ & Femoral and tibial \\
\hline
\end{tabular}

(*) H, D and $S$ refer respectively to hypertension, diabetes and smoking habit.

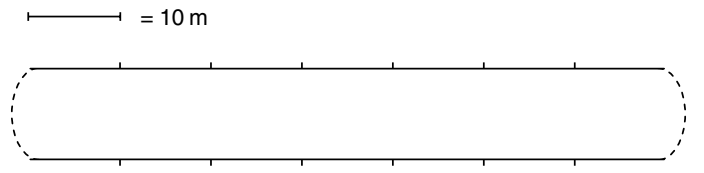

Fig 1. The course of the corridor test.

segments of $10 \mathrm{~m}$ each (Fig 1). During the test, an assistant accompanied the patient arm-in-arm and controlled the pace by checking the time interval of each fraction by stopwatch. A 5-min warm-up at a speed less than $1 \mathrm{~km} / \mathrm{h}$ preceded the test. The test started with the first $10 \mathrm{~m}$ section covered in a time ranging from 60 to $30 \mathrm{~s}$, depending on the clinical severity of the disease, the ABI values and the patient's age; the initial speed was therefore between 0.6 and $1.2 \mathrm{~km} / \mathrm{h}$. The time to cover the subsequent sections was reduced by $1-2 \mathrm{~s}$ for each section because of small accelerations imposed by the assistant at the beginning of each section. A second assistant registered the distance covered at the appearance of the claudication pain, promptly referred to by the patient and recorded the onset of minor symptoms, when present. The test was continued until pain did not allow for further increase in speed. At the end of the test, the split times were used to calculate the average speed maintained for each $10 \mathrm{~m}$ and a graph of distance over walking speed for each subject was constructed (Fig 2). The speed reached at the section of the course at which claudication occurred (ie, PTS) was identified. During the test, the subject's heart rate was monitored (Sport Tester, Polar Electro, Kempele, Finland) and the heart rate at PTS recorded.

\section{Repeatability of PTS Determination}

Repeatability of PTS determination was checked in 10 subjects ( 3 females, 7 males) who performed 2 walking tests within a few days under the same experimental conditions.

\section{Six-Month Rehabilitation Program}

A rehabilitation program based on walking workouts was carried out during autumn and winter (October to March). Body weight and percent body fat, determined by skinfold measurement, were recorded at the beginning and at the end of the study period.

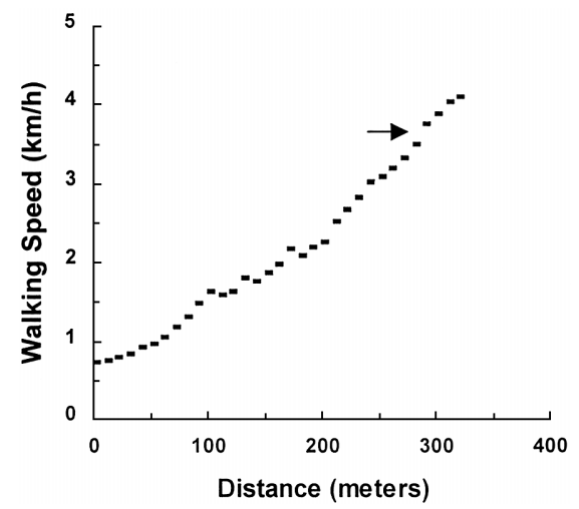

Fig 2. Increments in speed over distance for a patient performing the incremental walking test. Arrow indicates the speed at which claudication pain occurred (pain threshold speed, PTS).

All patients were asked to walk every other day, for at least $30 \mathrm{~min}$, at a speed $20-30 \%$ less than the PTS. Time intervals to cover a given distance at the assigned speed were given to each patient. A training heart rate range (10-15 beats/min less than the one registered at PTS) was also assigned and maintained through use of a heart rate monitor. Subjects were accompanied by one of the investigators during the first training session, and instructed how to keep the assigned walking velocity; they were also instructed in the use of the monitors. The subjects were asked to keep a record of training duration, distance covered and the appearance of any symptoms.

PTS and ABI were redetermined after 1, 3 and 6 months, and training intensities were adjusted according to the new PTS values recorded. The duration of the exercise sessions increased in all subjects from the initial $30 \mathrm{~min}$ to $60 \mathrm{~min}$ by the end of the 6 months.

\section{Statistical Analysis}

Data are expressed as mean \pm SD. Changes in PTS and $\mathrm{ABI}$ recorded during the 6-month period were evaluated with the analysis of variance (ANOVA) with repeated measures, and subsequently with the Newman-Keuls Multiple Comparisons test. A p value of 0.05 or less was considered statistically significant. 
Table 2 Average Values of Pain Threshold Speed (PTS) and Ankle Brachial Indexes (ABI) During Rehabilitation

\begin{tabular}{lrrrc}
\hline \hline & \multicolumn{4}{c}{ Months of rehabilitation } \\
\cline { 2 - 4 } & \multicolumn{1}{c}{0} & 1 & 3 & \multicolumn{1}{c}{6} \\
\hline \multirow{2}{*}{ PTS } & $3.9 \pm 1.40$ & $5.1 \pm 0.90^{*}$ & $5.7 \pm 1.10^{*}$ & $6.1 \pm 1.10^{* *}$ \\
$w A B I$ & $0.43 \pm 0.16$ & $0.51 \pm 0.17^{*}$ & $0.63 \pm 0.15^{*}$ & $0.72 \pm 0.15^{*}$ \\
$b A B I$ & $0.70 \pm 0.18$ & $0.77 \pm 0.17^{*}$ & $0.84 \pm 0.14^{*}$ & $0.88 \pm 0.14^{* *}$ \\
\hline
\end{tabular}

Values are mean $\pm S D$.

${ }^{*} p<0.05$ vs values of previous months, ${ }^{* *} p<0.05$ vs 0 and 1 month values.

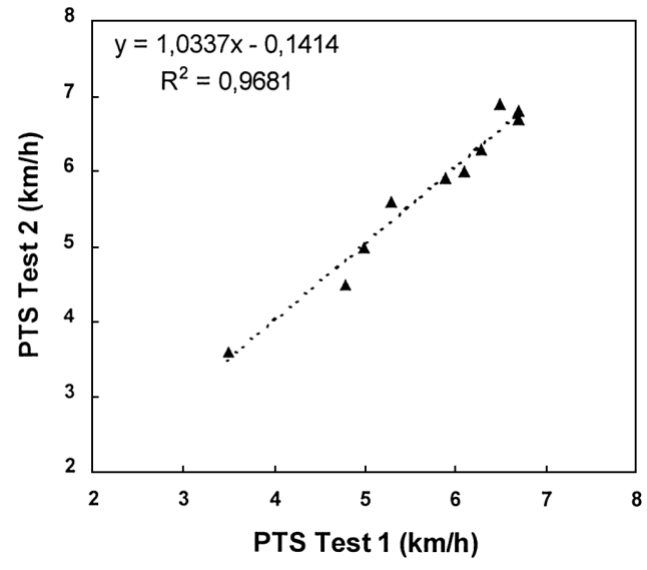

Fig 3. Repeatability of pain threshold speed (PTS) determination in 10 patients who underwent the incremental walking test within a few days.

\section{Results}

\section{Identification of the 'Pain Threshold Speed'}

Fig 1 shows the increments in speed over distance in a subject who underwent the new incremental walking test. The distance at the onset of pain and the corresponding speed are indicated. PTS was identified in all patients examined and the average PTS value (before rehabilitation) was $3.9 \pm 1.4 \mathrm{~km} / \mathrm{h}$ (Table 2$)$.

\section{Repeatability of PTS Determination}

In the 10 patients who performed 2 walking tests within 1 week, the PTS was $5.7 \pm 1.0 \mathrm{~km} / \mathrm{h}$ in the first test and $5.7 \pm 1.1 \mathrm{~km} / \mathrm{h}$ in the second (test-retest relationship: $\mathrm{R}=0.98, \mathrm{p}<0.001$; Fig 3 ). The heart rates at PTS were $106 \pm 11$ beats/min in the first test and $109 \pm 14$ beats/min in the second (test - retest relationship: $\mathrm{R}=0.96, \mathrm{p}<0.001$ ).

\section{Modifications in PTS and ABI During Rehabilitation}

Because the walking intensity chosen for rehabilitation was less than the pain threshold, none of the patients reported claudication or other symptoms during the training sessions for the 6-month period. All subjects completed the rehabilitation program. Average body weight was unmodified $(73.9 \pm 9.3 \mathrm{~kg}$ before, $73.0 \pm 8.7 \mathrm{~kg}$ after rehabilitation), with maximal individual variations ranging from a decrease of $8.3 \%$ to an increase of $3.8 \%$. Percent body fat was $23.8 \pm 7.9$ before and $23.7 \pm 6.7$ after rehabilitation. Smoking habits during the 6-month period were not modified.

Average values of PTS, wABI and bABI at 1, 3 and 6 months increased progressively and were significantly higher than at the start (Table2). The increments in ABI were because of increases in leg blood pressure, whereas the brachial blood pressure was unmodified. At the end of the rehabilitation period, improved blood flow in the obstructed limb areas was shown by echo-Doppler examination (data not shown). Average values for ABI recorded at $0,1,3$ and 6 months were correlated to average values for PTS (wABI to PTS: $r=0.96, p<0.05$; bABI to PTS: $\mathrm{r}=0.99, \mathrm{p}<0.01)$.

Average heart rates at PTS increased significantly during the 6-month rehabilitation period, being $98 \pm 12$ at the start and $109 \pm 10$ after 6 months $(\mathrm{p}<0.05)$.

\section{Discussion}

Intermittent claudication consists of a painful cramping that occurs in one or both legs while walking and its intensity depends on a discrepancy between oxygen supply, limited by the arteriopathy, and oxygen demand by the muscles involved in walking? It is a progressive functional limitation and causes a drastic reduction in mobility.

Evaluation of the degree of arteriopathy in patients with intermittent claudication is commonly based on the maximal walking distance allowed by the claudication reported by patients. Such evaluation has been examined and considered of little value in judging the real handicap of these patients? Currently used laboratory functional evaluations establish the walking distance covered in a given time ${ }^{4}$ or the claudication-free walking time and distance in standardized treadmill tests at constant or graded exercise intensity $1,2,5,6$ In treadmill testing the maximal walking distance and time of the subject are also considered.

We have evaluated the degree of peripheral leg arteriopathy by means of an incremental walking test designed to determine the maximal velocity that intermittent claudication patients can reach in the absence of pain (pain threshold speed [PTS]). This test can also be carried out on a motorized treadmill, which allows easily standardized conditions. However, the walking conditions of the corridor test (a flat and non-moving surface) were preferred by patients because they more closely resembled natural walking. The low starting speed $(\leq 1.2 \mathrm{~km} / \mathrm{h})$ prevented the early appearance of claudication pain in all patients, including those with severe arteriopathy. The small speed increments $(0.1-0.2 \mathrm{~km} / \mathrm{h})$ allowed for the identification of a precise speed at claudication. As indicated by the correlation found in a test-retest experiment, PTS values determined within a week are constant, which demonstrates that patients are able to identify precisely the onset of intermittent claudication pain and that the exercise intensity at which the symptom appears does not change significantly within a short time.

At the onset of pain, oxygen supply does not completely satisfy muscle requirements? which can lead to a local activation of the anaerobic lactacid mechanisms. In accordance with this hypothesis, a moderate but significant increase in 
blood lactate concentration was found at PTS in 6 of the present patients (lactate at rest $1.4 \pm 0.5 \mathrm{mmol} / \mathrm{L}$; lactate at PTS $2.3 \pm 0.4 \mathrm{mmol} / \mathrm{L}, \mathrm{p}=0.013)$. In this respect PTS could be compared to the anaerobic threshold. Similarly, the training workouts at less than the PTS used in our rehabilitation program could be compared to walking programs at intensities below the lactate threshold, found to be adequate aerobic training stimulus in elderly sedentary subjects?

For this reason the information obtained from the incremental corridor test was used to set up walking rehabilitation programs based on home workouts slightly below the individual PTS values. Being tailored to the capabilities of each subject, these programs allowed relatively long symptom-free training sessions, easy to comply with by all patients.

It is well documented that walking rehabilitation programs improve the functional parameters of intermittent claudication patients.,5,6 Improvements in the echo-Doppler pattern, ABI values, claudication walking distance and PTS were also documented in the present patients following rehabilitation. The average increments in ABI and PTS were significantly correlated. It can be concluded that PTS is a sensitive parameter of performance variations in these subjects.

After rehabilitation, the self-paced walking speed maintained during training sessions $(<\mathrm{PTS})$ was between 3 and $6 \mathrm{~km} / \mathrm{h}$, analogous to the self-selected pace documented in healthy subjects of the same age? The efficacy of rehabilitation programs below the pain threshold compared with protocols beyond the pain threshold remains to be established.

The improvements in PTS averaged $56.4 \%$ (from 3.9 to $6.1 \mathrm{~km} / \mathrm{h}$ ), with greater increments in the patients with the lowest baseline PTS values and can be explained by the fact that these subjects trained at speeds closer to PTS than those with higher PTS values. The former may have reached conditions of greater local hypoxia, triggering a greater increase in aerobic power through increased mitochondria and respiratory enzymes of the muscles involved in the exercise, 10 The training program was probably also followed by enhanced muscle capillarization: an increase in the total number of muscle capillaries has been reported following 2 months of endurance training at submaximal intensity 10 The improvements of echo-Doppler pattern and
ABI, observed in all patients, were probably caused by increased collateral blood flow 11,12

\section{Acknowledgments}

This study was supported by a research grant (ex-60\%) of the University of Ferrara.

The authors thank Mrs Patricia Ennis assistance with the manuscript, and Mr Daniele Buzzoni and Mrs Elisabetta Mariotti for their skilled technical assistance.

\section{References}

1. Regensteiner JG, Gardner A, Hiatt WR. Exercise testing and exercise rehabilitation for patients with peripheral arterial disease: Status in 1997. Vasc Med 1997; 2: 147-155.

2. Gardner A, Skinner J, Cantwell B, Smith LK. Progressive vs single stage treadmill tests for evaluation of claudication. Med Sci Sports Exerc 1991; 23: $402-408$.

3. Watson CJ, Phillips D, Hands L, Collin J. Claudication distance is poorly estimated and inappropriately measured. Br J Surg 1997; 84: 1107-1109.

4. Montgomery PS, Gardner AW. The clinical utility of a six-minute walking test in peripheral arterial occlusive disease patients. $J \mathrm{Am}$ Geriatric Soc 1998; 46: 706-711.

5. Brandsma JW, Robeer BG, van den Heuvel S, Smit B, Wittens CH, Oostendorp RA. The effect of exercises on walking distance of patients with intermittent claudication: A study of randomized clinical trials. Phys Ther 1998; 78: 278-288.

6. Regensteiner JG. Exercise in the treatment of claudication: Assessment and treatment of functional impairment. Vasc Med 1997; 2: $238-242$.

7. Belman MJ, Gaesser GA. Exercise training below and above the lactate threshold in the elderly. Med Sci Sports Exerc 1991; 23: $562-$ 568.

8. Himann JE, Cunningham DA, Rechnitzer PA, Paterson DH. Agerelated changes in speed of walking. Med Sci Sports Exerc 1988; 20: $161-166$.

9. Bylund-Fellenius AC, Walker PM, Elander A, Holm S, Holm J, Schersten T. Energy metabolism in relation to oxygen partial pressure in human skeletal muscle during exercise. Biochem J 1981; 200: $247-255$.

10. Henriksson J, Hickner RC. Adaptations in skeletal muscle in response to endurance training. In: Harries M, Williams C, Stanish WD, Micheli LJ, editors. Oxford textbook of sports medicine. New York: Oxford; 1998: 48-52.

11. Weiss T, Fujita Y, Kreimeier U, Messmer K. Effect of intensive walking exercise on skeletal muscle blood flow in intermittent claudication. Angiology 1992; 43: 63-71.

12. Yang HT, Dinn RF, Terjung RL. Training increases muscle blood flow in rats with peripheral arterial insufficiency. J Appl Physiol 1990; 69: $1353-1359$. 